\title{
Editorial
}

\section{Discrete Dynamics in Supply Chain Management}

\author{
Tinggui Chen, ${ }^{1}$ Kai Huang, ${ }^{2}$ and Zhigang Jiang ${ }^{3}$ \\ ${ }^{1}$ School of Computer and Information Engineering, Zhejiang Gongshang University, Hangzhou 310018, China \\ ${ }^{2}$ DeGroote School of Business, DSB 404, McMaster University, Hamilton, ON, Canada L8S4M4 \\ ${ }^{3}$ Green Manufacturing Engineering and Operations Management Institute, Wuhan University of Science and Technology, Wuhan, \\ Hubei 430081, China
}

Correspondence should be addressed to Tinggui Chen; ctgsimon@mail.zjgsu.edu.cn

Received 24 March 2014; Accepted 24 March 2014; Published 4 June 2014

Copyright ( $\odot 2014$ Tinggui Chen et al. This is an open access article distributed under the Creative Commons Attribution License, which permits unrestricted use, distribution, and reproduction in any medium, provided the original work is properly cited.

Supply chain management (SCM) is considered as the integration of business processes in providing products or services to end customers by establishing a strategic alliance of all parties, which has become a critical means of adding value to products and increasing the company's competitive advantage. However, organizations in the supply chains in real world are always vulnerable to partial or complete disruptions. Various factors such as natural disasters, labor strikes, power outages, parts shortages, quality related recalls, transportation interruptions, and machine breakdowns can lead to performance fluctuations or even disruptions of business processes in organizations, which indicates that supply chain processes are dynamic, discrete, volatile, and unpredictable.

The main objective of this special issue is to present the original research and review articles on the latest theoretical and practical achievements that will contribute to the field of discrete and dynamics supply chain management, in all branches of management science.

The special issue received 52 high quality submissions from different countries all over the world. All submitted manuscripts have followed the same standard (peer-reviewed by at least three independent reviewers) as applied to regular ones to this journal. Due to space limit, only 21 papers could be published (acceptance ratio of $1: 2.5$ ). Inevitably, difficult decisions had to be made, and some high-quality submissions could not be included. The primary guideline was to demonstrate discrete dynamics in supply chain management. Besides, some novel research questions from different applications that are worth further investigation in the future are also included.
In the paper "Price and service competition of dualchannel supply chain with consumer returns," L. Ren et al. propose dual-channel supply chain models involving consumer returns policies. Also, the price and service competition between retail channel and direct channel is considered in the models. According to the models, they analyze the optimal decisions in both centralized and decentralized scenarios. Then they design a new contract, coordinate the dualchannel supply chain, and enable both the retailer and the manufacturer to be a win-win.

In the paper "Viability discrimination of a class of control systems on a nonsmooth region," $\mathrm{N}$. Zhao et al. consider the viability for both an affine nonlinear hybrid system and a hybrid differential inclusion on a region with subdifferentiable boundary. Based on the nonsmooth analysis theory, they obtain a method to verify the viability condition at a point, when the boundary function of the region is subdifferentiable and its subdifferential is convex hull of many finite points.

In the paper "Incentive contract in supply chain with asymmetric information," Y. Su et al. use principal-agent theory and the theory of regulation to design the contract to realize the maximization of principal's profit on the condition that the contract satisfies the participant and incentive conditions of agent. As a result, it is obvious that the contract achieves the goal of control. In addition, it also can be concluded that the amount of rent that the manufacturer can obtain is up to the value of his information and the condition of his resource.

In the paper entitled "A constraint programming method for advanced planning and scheduling system with multilevel structured products," Y. Peng et al. deal with the advanced 
planning and scheduling (APS) problem with multilevel structured products. A constraint programming model is constructed for the problem with the consideration of precedence constraints, capacity constraints, release time, and due date. A new constraint programming $(\mathrm{CP})$ method is proposed to minimize the total cost. This method is based on iterative solving via branch and bound. And, at each node, the constraint propagation technique is adapted for domain filtering and consistency check. Three branching strategies are compared to improve the search speed. The results of computational study show that the proposed $\mathrm{CP}$ method performs better than the traditional mixed integer programming (MIP) method. And the binary constraint heuristic branching strategy is more effective than the other two branching strategies.

In the paper "Multiobjective vehicle routing problem with route balance based on genetic algorithm," W. Zhou et al. propose a genetic algorithm to solve the biobjective vehicle routing problem with time windows simultaneously considering total distance and distance balance of active vehicle fleet. A new complex chromosome is used to present the active vehicle route. Through tournament selection, onepoint crossover, and migrating mutation operator, the solution of the problem is solved. In experiment on Solomon's benchmark problems, considering the total distance and distance balance, the results are improved in all classes of problems. According to the experimental results, the suggested approach is sufficient and the average GA performance is good.

In the paper entitled "The game analysis of manufacturers' political connections on product safety in supply chain: evidence from China," Z. Na and W. Fusheng study the political connections on product safety in supply chain. In market economy, information asymmetry exists throughout the entirety of supply chains that ought to ensure product safety. Due to the existence of game relations between the government and manufacturers in the aspects of product safety and regulation, the formation of market equilibrium depends on political connections between the government and manufacturers. Based on study and analyses of a static game model and a dynamic game model, this paper reveals that governments and manufacturers must use positive political connections to achieve product protection and supervision of safety throughout the supply chain. On the other hand, negative political connections lead to losses of both governmental credibility and social profits. This study indicates that inherent mechanism of political connections exists in the supply chain; it will help to enrich the theory of supply chain.

The paper of $\mathrm{H}$. Wang et al. entitled "Optimizing route for hazardous materials logistics based on hybrid ant colony algorithm" devises an improved hybrid ant colony algorithm (HACA) to deal with optimizing route for hazardous materials logistics (ORHML). To achieve the purpose of balancing risk and cost for route based on the principle of ACA that used to solve TSP, the improved HACA was designed. Considering the capacity of road network and the maximum expected risk limits, a route optimization model to minimize the total cost is established based on network flow theory. Improvement on route construction rule and pheromone updating rule was adopted on the basis of the former algorithm. An example was analyzed to demonstrate the correctness of the application. It is proved that improved HACA is efficient and feasible in solving ORHML.

In the paper entitled "System dynamics model for VMIङTPL integrated supply chains," G. Li et al. establish VMI-APIOBPCS II model by extending VMI-APIOBPCS model from serial supply chain to distribution supply chain. Then TPL is introduced to this VMI distribution supply chain, and operational framework and process of VMI\&TPL integrated supply chain are analyzed deeply. On this basis VMI-APIOBPCS II model is then changed to VMI\&TPLAPIOBPCS model and VMI\&TPL integrated operation mode is simulated. Finally, compared with VMI-APIOBPCS model, the TPL's important role of goods consolidation and risk sharing in VMI\&TPL integrated supply chain is analyzed in detail from the aspects of bullwhip effect, inventory level, service level, and so on.

In the paper entitled "A location-allocation model for seaport-dry port system optimization," $\mathrm{X}$. Feng et al. construct a location-allocation model for the regional seaport-dry port network optimization problem and develop a greedy algorithm and a genetic algorithm to obtain its solution. This model is applicable to situations under which the geographic distribution of demand is known. A case study involving configuration of dry ports near the west bank of the Taiwan Strait is conducted, and the model is successfully applied.

In the paper entitled "An inventory controlled supply chain model based on improved BP neural network," Wei He applies the improved BP neural network model to predict the inventory level of an automotive parts company. The results show that the improved algorithm not only significantly exceeds the standard algorithm but also outperforms some other improved BP algorithms both on convergence rate and prediction accuracy.

In the paper entitled "Tourist behavior pattern mining model based on context," D.-s. Liu and S.-j. Fan take the context into consideration and propose an analyzed method to the tourist based on the context. Firstly, they analyze the context which influences the tourist behavior patterns, select the main context factors, and construct the tourist behavior pattern model based on it. Then, they calculate the interest degree of the tourist behavior pattern and mine out the rules with high interest degree with the association rule algorithm. At last, an experiment is given so as to show the feasibility and effectiveness of their method.

In the paper entitled "Coordination in the decentralized assembly system with dual supply modes," X. Guan and M. Liu investigate a decentralized assembly system that consists of one assembler and two independent suppliers, wherein one supplier is perfectly reliable for the production, while the other generates yield uncertainty. Facing the random market demand, the assembler has to order the components from one supplier in advance and meanwhile requires the other supplier to deliver the components under VMI mode. They construct a Nash game between the supplier and the assembler so as to derive their equilibrium procurement/production strategies. The results show that the channel's performance is highly undermined by the decentralization between players 
and also the combination of two supply modes. Compared to the centralized system, they propose an advance payment contract to perfectly coordinate the supply chain performance. The numerical examples indicate some management implications on the supply mode comparison and sensitivity analysis.

In the paper "Dynamic pricing and supply coordination with reimbursement contract under random yield and demand," G. Li et al. study the dynamic pricing and supply chain coordination in a decentralized system that consists of one supplier and one manufacturer, in which both the market demand and production yield are stochastic. They show that the centralized expected profit is jointly concave in the production quantity and order quantity when the price is ex ante selected. They also derive the equilibrium strategies in the decentralized system and prove that the entire profit of supply chain is inevitably lower than that under centralized system. Based on this, the authors propose a reimbursement contract to coordinate the decentralized supply chain so as to achieve the maximized profit. It is worth mentioning that, under reimbursement contract, the equilibrium production and order quantities are irrelevant to the manufacturer's risk sharing coefficient but are only determined by the supplier's risk sharing coefficient.

In the paper "Research on self-organization in resilient recovery of cluster supply chains," L. Geng et al. explore dealing with high-risk and low-probability disruptions. First, the paper describes the representation method of cluster supply chain resilience. Second, a cluster supply chain network structure generation model is proposed. And based on cascading effect model, it makes analysis of dynamic evolution process when cluster supply chain failure happens. Then it focuses on the self-organization characteristic, which contributes to cluster supply chain emergence of overall resilient recovery through local self-organization reconstruction behavior. They also make theoretical analysis of cluster supply chain network characteristics and its effect on the resilience, which helps to illustrate that the root of vulnerability lies in cascading failure while self-organization is the key to resilient recovery. Besides, with the study of selforganization characteristic, it provides theoretical guidance for local control and further achievement of overall resilient optimization.

In the paper entitled "Optimal acquisition and inventory control for a remanufacturing system," Z. Jiang et al. propose a method for optimal acquisition and inventory control of a remanufacturing system. The method considers three inventories, one for returned item and the other for serviceable and recoverable items. Taking the holding cost for returns and recoverable and remanufactured products, remanufacturing cost, disposal cost, and the loss caused by backlog into account, the optimal inventory control model is established to minimize the total costs. Finally, a numerical example is provided to illustrate the proposed methods.

In the paper "Two-level credit financing for noninstantaneous deterioration items in a supply chain with downstream credit-linked demand," Y. He and H. Huang assume that the items have the property of noninstantaneous deterioration and the demand is a function of downstream credit. Then, an EOQ model for noninstantaneous deterioration is built based on the two-level financing policy. The purpose of this paper is to maximize the total average profit by determining the optimal downstream credit period, the optimal replenishment cycle length, and the optimal ordering quantity per cycle. Useful theorems are proposed to characterize the method of obtaining the optimal solutions. Based on the theorems, an algorithm is designed, and numerical tests and sensitive analysis are provided. Lastly, according to the sensitive analysis, managerial insights are proposed.

In the paper "Location optimization of multidistribution centers based on low-carbon constraints," $\mathrm{P}$. Zhao et al. analyze the necessity of industrial carbon dioxide emission cost internalization in four aspects and build a model for multidistribution centers location in effort to reduce carbon footprint that can provide optimized strategy support for decision makers and logistic operators. Numerical examples are presented to illustrate the feasibility and effectiveness of the models.

In the paper "Simulation research of space-time evolution of emergency logistics network reliability based on complex network theory," L. Huang et al. propose the conception and evaluation indexes of emergency logistics network connecting reliability to construct evaluation index system of complex network reliability and describe these indexes quantitatively to evaluate the network connecting reliability. Moreover, the network topological model and the simulation methods of reliability measurement when the network is under attack are present. Finally, the authors take three classical emergency logistics networks as examples, and through emulation analysis they obtain the connecting reliability changing situation of these three networks under random attack, the changing curve of the ratio of effective demand nodes, and emergence supply mileage of emergency logistics network with the same network density but different forms and then evaluate the emergency logistics network connecting reliability. This can provide references for the designing of emergency logistics network with high reliability and analysis means for research in other fields.

In the paper entitled "Risk-averse suppliers' optimal pricing strategies in a two-stage supply chain," risk-averse suppliers' optimal pricing strategies in two-stage supply chains under competitive environment are discussed. The suppliers in this paper focus more on losses as compared to profits, and they care about their long-term relationship with their customers. R. Shen et al. introduce for the suppliers a loss function, which covers both current loss and future loss. The optimal wholesale price is solved under situations of risk neutral, risk averse, and a combination of minimizing loss and controlling risk, respectively. Besides, some properties and relations among these optimal wholesale prices are given as well. A numerical example is given to illustrate the performance of the proposed method.

In the paper "Personal recommendation using a novel collaborative filtering algorithm in customer relationship management," C. Xu constructs a user recommendation model containing a new method to compute the similarities among users on bipartite networks. Different from other standard similarities, he considers the influence of each object node 
including popular degree, preference degree, and trust relationship. Substituting these new definitions of similarity for the standard cosine similarity, the author proposes a modified collaborative filtering algorithm based on multifactors (CF-M). Detailed experimental analysis on two benchmark datasets shows that the CF-M is of high accuracy and also generates more diversity.

In the paper "Collaborative policy of supply-hub for assemble-to-order systems with delivery uncertainty," G. Li et al. build a collaborative replenishment model in the assembly-to-order system based on supply-hub under delivery uncertainty, transform the original model into a onedimensional optimization problem appropriately, and then calculate the optimal assembly quantity of the manufacture and the optimal order points of various component suppliers. In order to enable collaborative replenishment, penalties and bonus mechanisms are proposed for supplyhub to coordinate various component suppliers. Based on the analysis in detail, they can conclude that when the penalty coefficient is very large, the suppliers will do their best to deliver on time, and when the reward of the supplier's ontime delivery is great enough, the suppliers will avoid delay delivery. The numerical analyses also show that penalty and bonus mechanisms can significantly improve the supplier's initiative of collaborative replenishment, thus improving the service level in assemble-to-order systems. This paper also provides a theoretical basis as well as useful guidance to the practice of collaborative replenishment in assemble-to-order systems based on supply-hub under delivery uncertainty.

The study of discrete dynamics in supply chain management is still in its early stage. This special issue demonstrates the theoretical and practical importance of further studies in discrete dynamics in supply chain management.

\section{Acknowledgments}

We would like to express our gratitude to all of the authors for their contributions and the reviewers for their effort providing valuable comments and feedback. We hope this special issue offers a comprehensive and timely view of the area of discrete dynamics in supply chain management and that it will offer stimulation for further research. The work is also supported by the National Natural Science Foundation of China (51205295) and Wuhan Youth Chenguang Program of Science and Technology (2014070404010214).

Tinggui Chen

Kai Huang

Zhigang Jiang 


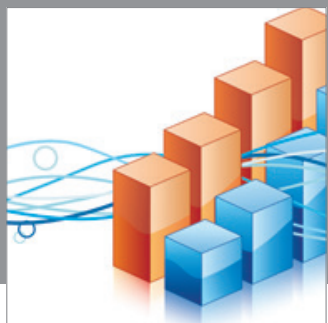

Advances in

Operations Research

mansans

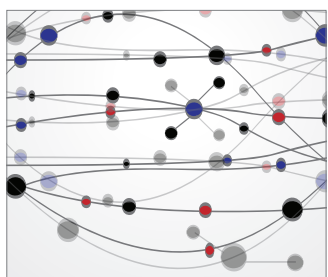

The Scientific World Journal
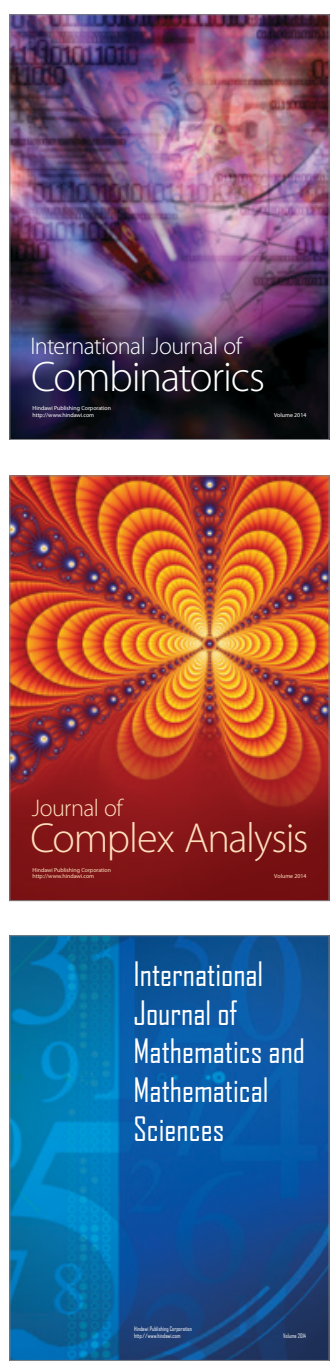
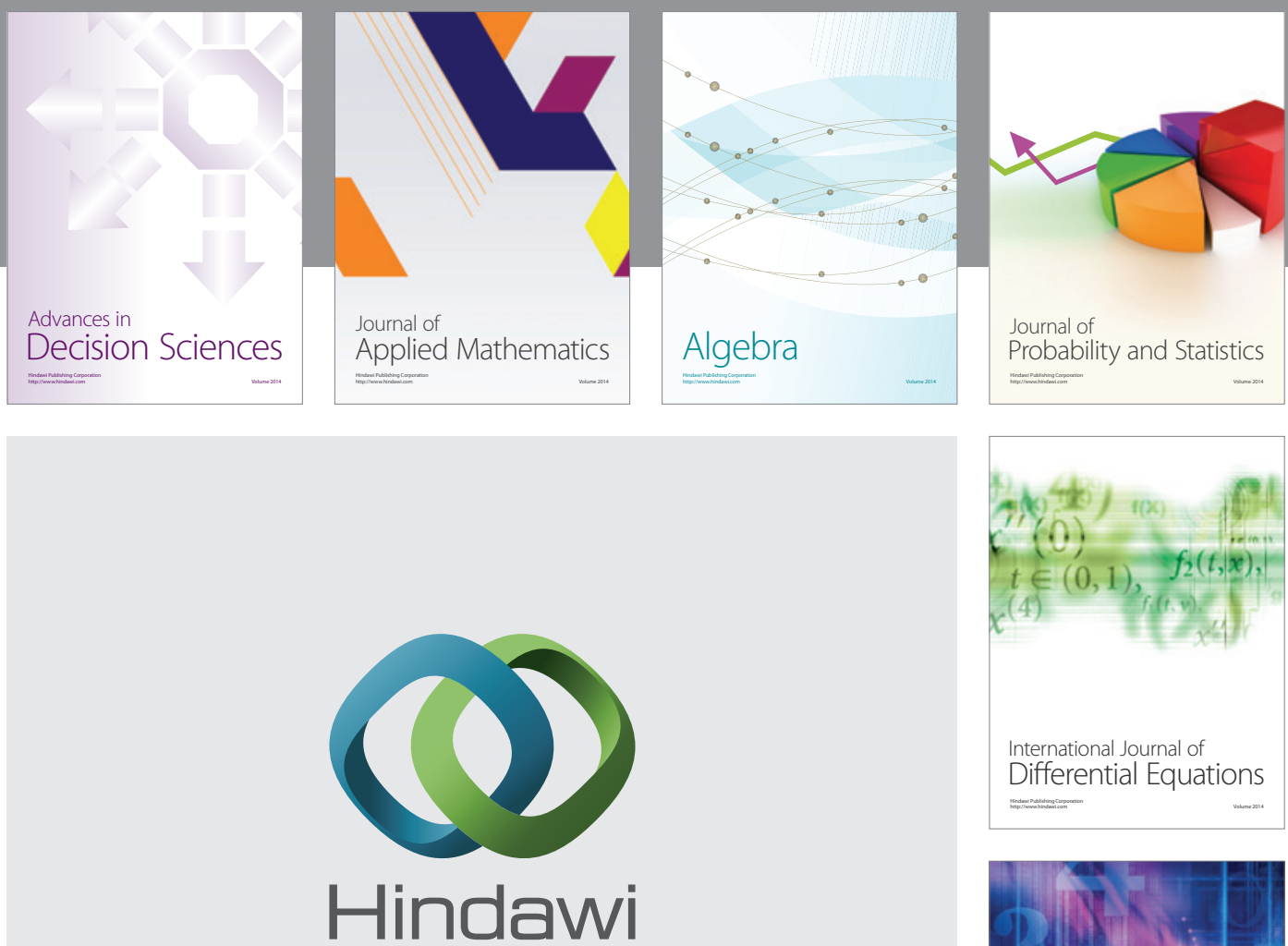

Submit your manuscripts at http://www.hindawi.com
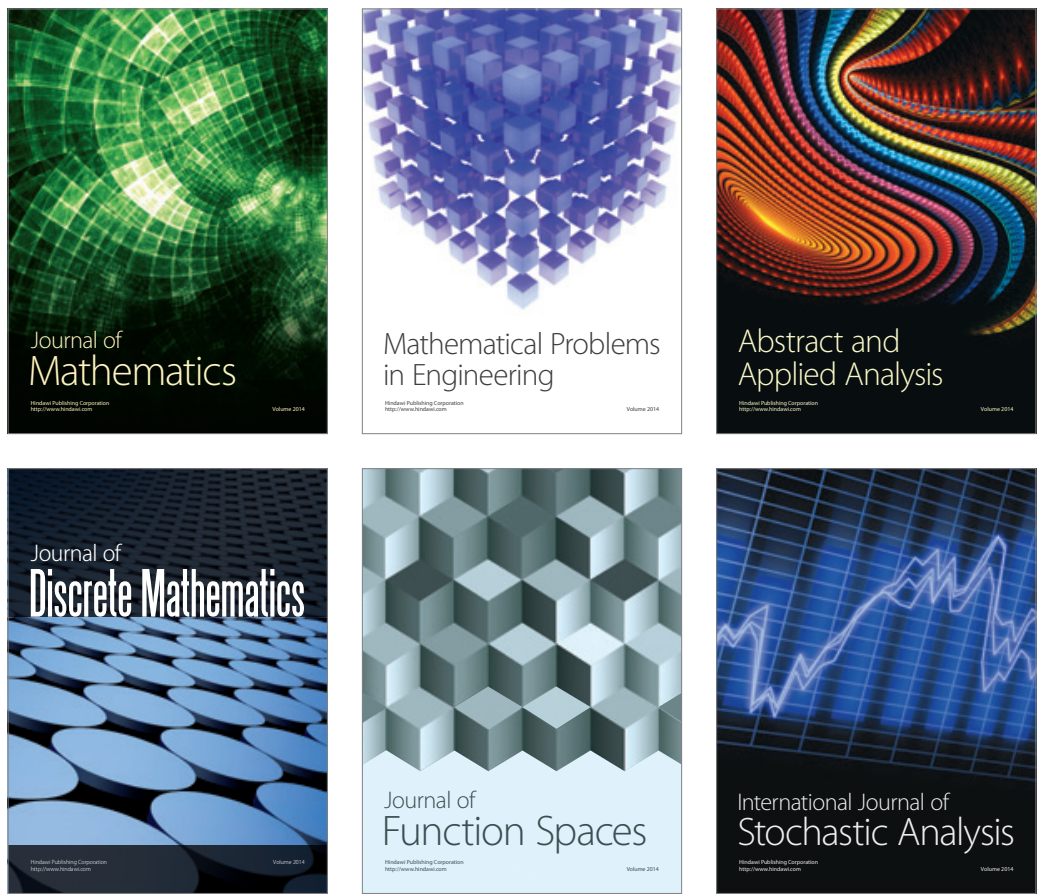

Journal of

Function Spaces

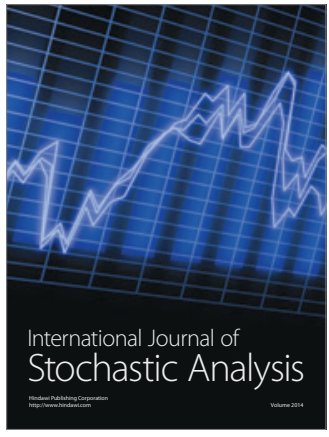

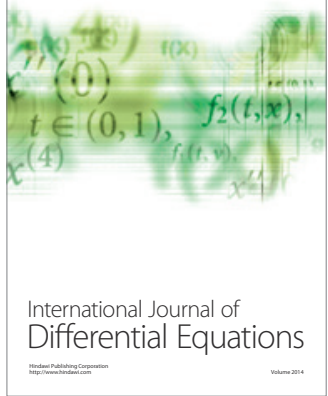
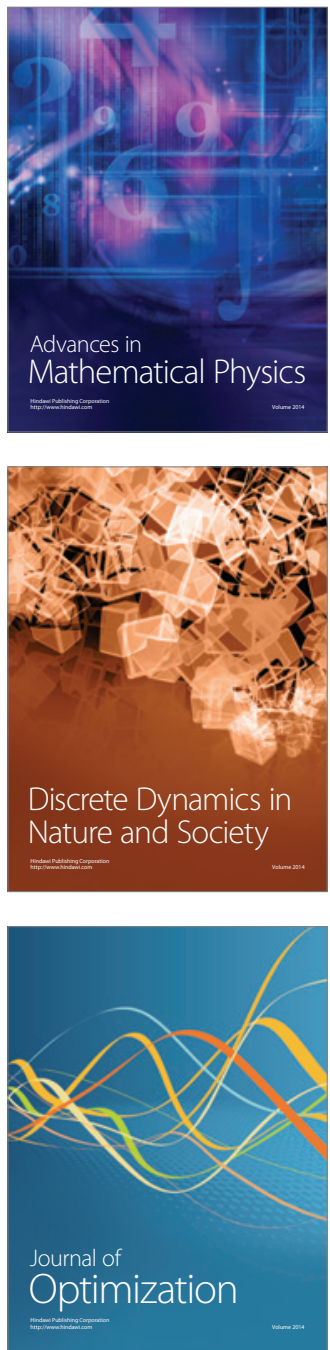